\title{
Spin-guided artillery shells autopilot design and decoupling control
}

\author{
Le Zhao, Xugang Wang \\ Nanjing University of Science and Technology, China \\ 617641889@qq.com
}

Keywords: spin-guided artillery shells; feed-forward compensation; decoupling control

\begin{abstract}
In this paper, a delay for the guided artillery shells in response to the instruction control mechanism designed to compensate autopilot elastomer damping. For the cross-coupling effects generated periodically roll pitch and yaw between channels, combined with PI controller design decoupling control algorithm, establish missile mathematical model solved for the transfer function of pitch and yaw direction, determine the damping range combined with the root locus method, design autopilot simulation, use PID control combined with feedforward compensator design Guided Projectile decoupling control system. This approach simplifies the controller, improve the quality of the transition process, improve maneuverability elastomer itself and realize the strong coupling system guided projectile decoupling. After numerical analysis, and in the case of strong coupling perturbation parameters, autopilot and control methods have rapid response capability and robustness characteristics and achieve decoupling, the system meet the system performance requirements.
\end{abstract}

\section{Introduction}

Guided projectile is an important direction of development of weapons systems, however, it is a strongly coupled nonlinear multivariable complex system. At home and abroad to roll shells decoupling control conducted in-depth research. The initial decoupling control method is an approximate method rotary shells lateral movement based on linear system theory[1], then Yuan Zaihuai [2] use coupled in series compensator decoupling method. After Yanxiao Yong et al [3-4] by analysing the instruction compensation decoupling, multivariable frequency domain limitations and effectiveness of decoupling, designed the pre-compensation methods. Ruisheng Sun et al [5] using a matrix of mathematical methods decoupling compensation.

In this paper, the root locus method [6] strike damping, design damping control loop to improve the dynamic response characteristics of projectile, determining the degree of coupling systems, design the feed forward compensation decoupling of pitch and yaw channels, which use the PID control algorithm, combined with feed-forward compensator design a guided projectile decoupling control system. Through simulation, the designed controller can parameter perturbations and the case of strong coupling, to achieve fast and accurate tracking of instruction.

\section{Guided artillery shells kinetic equation}

\subsection{Dynamics model.}

Guided artillery shells motion model is very complex nonlinear time-varying model for the study brought greater inconvenience. To facilitate the analysis and applications, we need to be linearized equations of motion. After the introduction of the assumptions of linear kinetic equations are as follows 


$$
\begin{aligned}
& \left\{\begin{array}{l}
\frac{\mathrm{d}^{2} \Delta \vartheta}{d \mathrm{t}^{2}}-a_{22} \frac{d \Delta \vartheta}{d t}-a_{24} \Delta \alpha+a_{27}^{\prime} \Delta \beta-a_{28}^{\prime} \frac{d \Delta \psi}{d t}=a_{25} \Delta \delta_{z} \\
\frac{\mathrm{d}^{2} \Delta \psi}{d \mathrm{t}^{2}}-a_{22} \frac{d \Delta \psi}{d t}-a_{24} \Delta \beta-a_{27}^{\prime} \Delta \alpha+a_{28}^{\prime} \frac{d \Delta \vartheta}{d t}=a_{25} \Delta \delta_{y} \\
\frac{d \Delta \theta}{d t}-a_{34} \Delta \alpha=a_{35} \Delta \delta_{z} \\
\frac{d \Delta \psi_{v}}{d t}-a_{34} \Delta \beta=a_{35} \Delta \delta_{y} \\
-\Delta \vartheta+\Delta \theta+\Delta \alpha=0 \\
-\Delta \psi+\Delta \psi_{v}+\Delta \beta=0
\end{array}\right. \\
& a_{i j}, b_{i j} \text { are aerodynamic parameters, each of which specific definition in Ref. [7] }
\end{aligned}
$$

\subsection{Establish guided artillery shells coupling transfer function.}

Disturbance of shells equations of motion (1) Laplace transform, the original function becomes image function, after finishing can be written in the form of a matrix equation

$$
\left[\begin{array}{cccccc}
s\left(s-a_{22}\right) & -a_{24} & a_{27} & -a_{28} s & 0 & 0 \\
a_{28} s & -a_{27} & -a_{24} & s\left(s-a_{22}\right) & 0 & 0 \\
0 & -a_{34} & 0 & 0 & 0 & s \\
0 & 0 & -a_{34} & 0 & s & 0 \\
-1 & 1 & 0 & 0 & 0 & 1 \\
0 & 0 & 1 & -1 & 1 & 0
\end{array}\right]\left[\begin{array}{c}
\Delta \vartheta(s) \\
\Delta \alpha(s) \\
\Delta \beta(s) \\
\Delta \psi(s) \\
\Delta \psi \psi_{v}(s) \\
\Delta \theta(s)
\end{array}\right]=\left[\begin{array}{c}
a_{25} \\
0 \\
a_{35} \\
0 \\
0 \\
0
\end{array}\right] \delta_{g}+\left[\begin{array}{c}
0 \\
a_{25} \\
0 \\
a_{35} \\
0 \\
0
\end{array}\right] \delta_{\psi}
$$

Without considering the impact of cross-linking, input system is equivalent to the pitch and yaw rudder angle $\delta_{\vartheta}, \delta_{\psi}$ the output pitch and yaw angle $\vartheta$ and $\psi$

$$
\left[\begin{array}{l}
\vartheta \\
\psi
\end{array}\right]=G_{1}\left[\begin{array}{l}
\delta_{\vartheta} \\
\delta_{\psi}
\end{array}\right]
$$

\subsection{Autopilot design.}

Closed-loop transfer function typical feedback control system is

$\frac{X_{o}(s)}{X_{i}(s)}=\frac{G(s)}{1+G(s) H(s)}$

Converted to the form of the Bernoulli equation can be written as

$G(s) H(s)=\frac{K^{*} A_{z 1} e^{j \theta_{z 1} \cdots} A_{z m} e^{j \theta_{z m}}}{A_{p 1} e^{j \theta_{p}} \cdots A_{p n} e^{j \theta_{p n}}}$

Root locus method can be obtained in damping K's value, closed-loop transfer function block diagram of the system are as follows

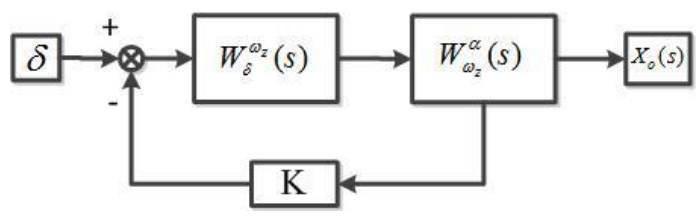

\section{Decoupling control design}

\subsection{Coupled analysis.}

References relative gain judging the coupling system, for steady-state matrix expression dual input and output of the system is

$$
\left[\begin{array}{l}
y_{1} \\
y_{2}
\end{array}\right]=\left[\begin{array}{ll}
k_{11} & k_{12} \\
k_{21} & k_{22}
\end{array}\right]\left[\begin{array}{l}
x_{1} \\
x_{2}
\end{array}\right]
$$

\subsection{Decoupling controller design.}

Decoupling control principle. On the basis of the invariance principle, feedforward control is working according to the principle of compensation according to changes in the disturbance or setpoint. Principle shown in Fig. 1 


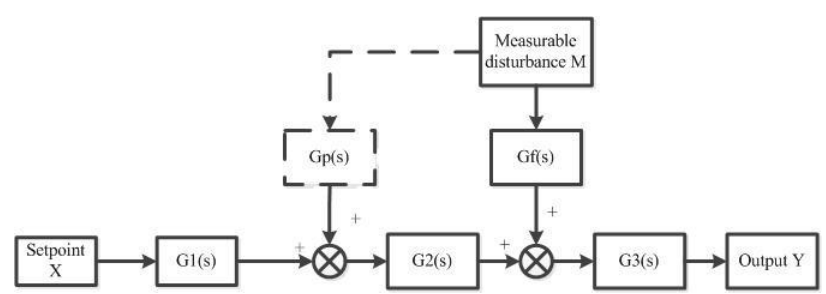

Figure 1. Feed-forward control schematic.

The solid line in Figure 1 is the original system configuration diagram, the dotted line is a part of the introduction of feedforward compensation, which $G_{f} G_{3}$ is an interference channel transfer function; $G_{P}(s)$ feedforward compensator function; $G_{2}(s) G_{3}(s)$ control channel transfer function; $G_{1}(s)$ system control link. Therefore, the system output is obtained

$$
Y=X \cdot G_{1} G_{2} G_{3}+M\left(G_{P} G_{2}+G_{f}\right) G_{3}
$$

Designing the feed forward compensation decoupling control system. The decoupling process transfer function of each channel is not the original $G_{i j}(s)$, so choose some $G_{P i j}(s)=1$ complete decoupling. transfer function model corresponding to the projectile is expressed as

$$
G_{p}(s)=\left[\begin{array}{cc}
G_{P 11}(s) & G_{P 12}(s) \\
G_{P 21}(s) & G_{P 22}(s)
\end{array}\right]=\left[\begin{array}{cc}
G_{11}(s) & G_{12}(s) \\
G_{21}(s) & G_{22}(s)
\end{array}\right]\left[\begin{array}{cc}
G_{11}(s) & 0 \\
0 & G_{22}(s)
\end{array}\right]=\left[\begin{array}{cc}
G_{11}(s) G_{22}(s) & -G_{22}(s) G_{12}(s) \\
-G_{11}(s) G_{21}(s) & G_{11}(s) G_{22}(s)
\end{array}\right] /\left[G_{11}(s) G_{22}(s)-G_{21}(s) G_{12}(s)\right]
$$

Select $G_{P 11}(s)=G_{P 22}(s)=1$, a block diagram of decoupling control system [8] as follows, tracking control system which instruction is pitch rudder angle $\delta_{\theta}$ and yaw rudder angle $\delta_{\psi}$, the system output is the pitch angle $\vartheta$ and yaw angle $\psi$ :

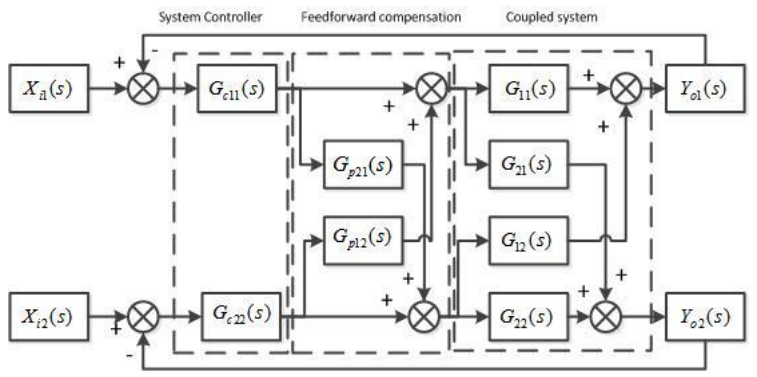

Figure 2. Feedforward compensation method decoupling control system structure.

Design PI controller, an improved Ziegler-Nichols method [9] for parameter tuning. The output signal of the controller is

$$
u(t)=K_{p} e(t)+\frac{K_{p}}{T_{i}} \int_{0}^{t} e(t) d t
$$

In summary, by the formula (8) and (9) together form a feed-forward compensation decoupling PID control system, which implements the coupling system for dual-input dual-output decoupling.

\section{Example simulation}

Now with a guided artillery shells numerical simulation example. Design damping control loop and autopilot structure and give it a unit step command signal, observe the corresponding rocking angular velocity, angle of attack and other output conditions, specifically as shown in Figure 3.
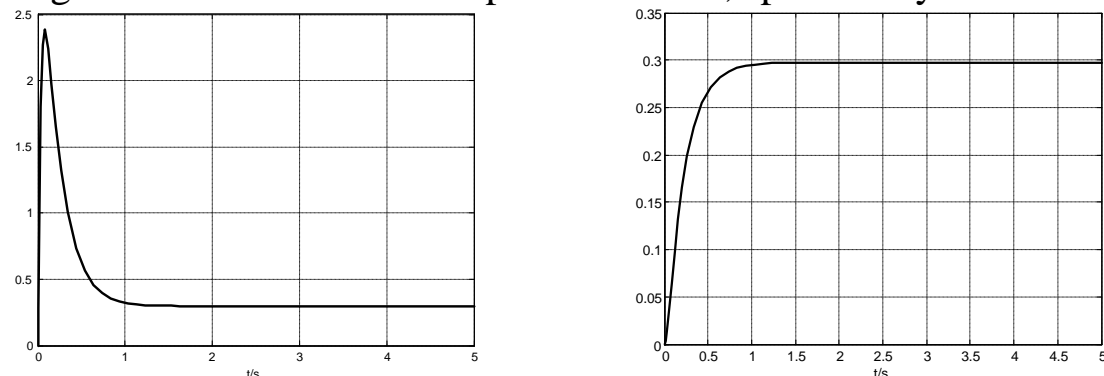

Figure 3. Damping circuit swing angular velocity and attack rate response 
From the above chart, the oscillation angle of attack does not exist, it does not overshoot and rapid response.Establish decoupling control system, set the spin speed $8 \mathrm{r} / \mathrm{s}$, respectively input step instruction $4^{\circ}$ yaw angle and the pitch angle of $5^{\circ}$, simulating while maintaining the reference parameters in the force and moment coefficients and perturbation of $\pm 30 \%$,

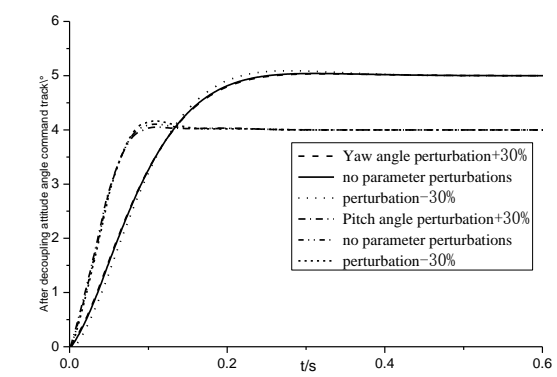

Figure 4. Instruction traces of the Decoupling Control System

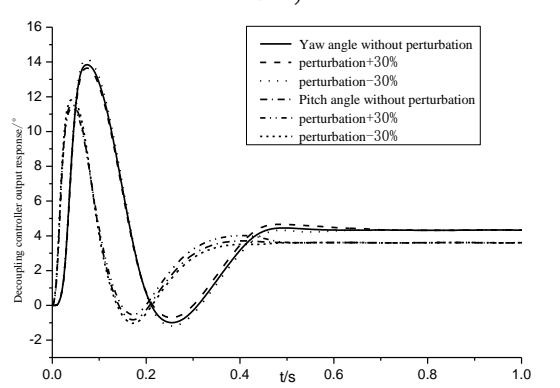

Figure 5. The controller step response curve

\section{Conclusion}

In this paper, the dynamic characteristics of the spin shells damping compensation itself, constitute autopilot to improve the quality of its transition. And analysis of a method for decoupling guided artillery shells cross-coupling effect generated periodically roll pitch and yaw between channels. Through simulation, decoupling control system is to verify that it enables accurate tracking of instructions when there is a strong coupling and parameter perturbations, and with rapid response capability and robustness characteristics, it can meet the actual needs of the project.

\section{References}

[1] Yuan Zihuai. An Approximate Method of Decoupling Longitudinal and Lateral Motions of Rolling Missiles.[J]. Beijing INST Technol, 1989, 9 (2): 41-50.

[2] Fu Yu, Yang Jun. Double Channel Autopilot Decoupling Control for Guided Projectile[J]. Computer Simulation, 2009, 26(10): 48-51.

[3] Yan Xiaoyong. Dynamic Stability and Control of rotating bomb [D]. Beijing Institute of Technology doctoral dissertation, 2010.

[4] Yan Xiaoyong, Yang Shuxing, Zhang Cheng. Decoupling Technique for a Class of Rolling Missile [J]. Journal of Ballistics, 2009, 21(4): 17-20.

[5] Sun Ruisheng, Xue Xiaozhong, Shen Jianping. Decoupling Control and Simulation Between Pitching and Yawing Channelsfor Small Diameter Bomb [J]. Journal of Ballistics, 2010, 22(2): 35-38.

[6] Zhang Baili. The Design of Series Leading Corrector Based on Root Locus [J]. Journal of Changchun Normal University(Natural Science),2012,03:63-66.

[7] Qian Xinfang, Lin Ruixiong, Zhao Yanan. Missile flight Mechanics [M]. Beijing Institute of Technology Press, 2006, 60-74.

[8] Yin Meilan. Research on MIMO decoupling internal model control [D]. Beijing University of Chemical Technology, 2006.

[9] Xu Jianming. PID Controller and Its Design Methods [D]. Zhejiang University of Technology, 2003. 